\title{
Generación K: Oportunidades y desafíos
}

\section{Generation K: Opportunities \& Challenges}

\author{
Javier Iturralde de Bracamonte
}

Universidad Politécnica de Valencia, jitudeb@doctor.upv.es

\begin{abstract}
Resumen
La industria cultural viene cambiando mucho en los últimos años, no sólo en lo que respecta a sus procesos de producción y distribución, sino también los hábitos, los gustos y las prácticas culturales. No obstante, la COVID-19 acelera una transformación digital crucial y más que necesaria en el sector del arte y de los museos, lo que representa a priori una gran oportunidad para el sector. Debido a las actividades implementadas y a los ajustes realizados en lo relativo a los recursos económicos y humanos, más del $40 \%$ de los museos encuestados notaron un aumento en las visitas online (NEMO, 2020). El objetivo principal de esta comunicación es analizar si ha aumentado el interés en el consumo de contenidos culturales, mediante canales digitales, por parte de los jóvenes $y$, en especial, por parte de la generación $K$, quienes en el año 2021 tendrían entre 13 y 21 años de edad. Otro de los objetivos es analizar algunas de las características y hábitos de consumo de la generación $K$, así como analizar qué tácticas y estrategias pueden ser más eficientes a la hora de atraer, fidelizar y capitalizar de manera sostenible a este público especifico y entender si realmente podría existir una oportunidad para los museos. Se trata de un trabajo de reflexión en el que se han empleado datos estadísticos, asi como otras fuentes secundarias pertinentes, y se han analizado proyectos y campañas desarrolladas por distintos museos. Mediante el análisis de dichos estudios se ha realizado una original aportación de los usos y costumbres actuales de la generación K en el consumo de arte y museos.
\end{abstract}

Palabras clave: comunicación, marketing, museos, generación $K$, redes sociales

\footnotetext{
Abstract

The cultural and creative industries have been changing in recent years, not only in terms of its production and distribution, but also in terms of its habits, trends and practices. However, COVID-19 has accelerated a digital
} 
transformation within the art and museum sector, representing a potential great opportunity. Due to the new activities online implemented plus the adjustments regarding financial and human resources, more than $40 \%$ of the museums that were surveyed noted an increase regarding its online visits (NEMO, 2020). The main objective of this communication is to analyze if the interest in the consumption of online cultural content has increased, by young people and, more especially, by generation K, (13-21 years old). Another objective is to analyze some of the characteristics and consumption habits of generation $K$, as well as to analyze what tactics and strategies can be more efficient when it comes to attracting, retaining and capitalizing on this specific audience and understanding whether they really there could be an opportunity for museums. This is a work of reflection in which statistical data has been used, as well as other pertinent secondary sources. Museum campaigns have been also analyzed. Through the analysis of these studies, an original contribution has been made of the current uses and habits of generation $K$ regarding the consumption of art and museums.

Keywords: communication, marketing, museums, generation $K$, and social media. 


\section{Introducción}

La crisis del coronavirus ha impactado en todas las dimensiones de nuestras vidas: la salud pública, el sistema laboral, la interacción social, el debate político, el uso del espacio público, la economía y el medio ambiente, entre otros aspectos. En lo que se refiere al arte y a la cultura la COVID-19 está afectando de manera muy directa y grave en todos los ámbitos. Se estima que el $90 \%$ de los aproximadamente 60.000 museos del mundo se han visto obligados a cerrar total o parcialmente sus puertas durante, al menos, tres meses, y a suspender temporalmente sus actividades públicas con el fin de garantizar la salud de visitantes, usuarios y trabajadores (UNESCO, 2020).

Independientemente de su tamaño, ubicación o situación, los museos se enfrentan actualmente a retos difíciles, que incluyen la protección de sus colecciones, la garantía de la seguridad y la salud del personal, así como el mantenimiento del compromiso con su público, momento en el que muchos de sus contenidos se trasladan, o amplían, a sus páginas web y redes sociales respectivas. A esto se suman las pérdidas millonarias ocasionadas por los meses de cierre, pero también por las pocas visitas in situ, lo cual provoca no solo una menor cantidad de entradas vendidas sino también una disminución considerable de los ingresos producidos por el consumo en el interior de estos. En promedio, el sector prevé perder un 36,5\% de sus ingresos en 2020, según el informe Urgente de la Fundación Contemporánea. Por otro lado, una encuesta realizada por Americans for the Arts estima que las pérdidas para las instituciones artísticas sin ánimo de lucro en Estados Unidos es ya de 3.200 millones de euros, y vaticina que un 33\% de los museos del país están en riesgo de cierre permanente. En el caso de España, el Museo Nacional del Prado, el Museo Nacional Centro de Arte Reina Sofía y el Museo Nacional Thyssen-Bornemisza han registrado una caída del $81 \%$ de las visitas este verano pasado (junio, julio y agosto 2020), respecto al mismo periodo del año anterior, lo que representa un total de 269.563 visitantes, cifra inferior a los 1,4 millones que acudieron en 2019. Este descenso se debe, por un lado, a las restricciones de aforo impuestas para evitar contagios y la amplificación del virus y, por otro, a una realidad que lamentablemente va a ser cada vez más frecuente, y se trata de una menor capacidad económica de la población más vulnerable como efecto colateral de esta pandemia. Asimismo, no existe una confianza plena en la vuelta a los recintos culturales y, por lo tanto, el regreso se está dando de manera gradual. Según Ferrán López, CEO de TekneData, esta transición tomará más de 24 meses. 


\section{Objetivos y Metodología}

El objetivo principal de esta comunicación es analizar si efectivamente ha aumentado el interés en el consumo de contenidos culturales, principalmente mediante canales digitales, por parte de los jóvenes y en especial por parte de la generación $\mathrm{K}^{1}$, quienes en el año 2021 tendrían entre 13 y 21 años de edad. Otro de los objetivos es analizar sus características y hábitos de consumo en el contexto del mundo del arte y de los museos, mediante el análisis de informes, estrategias y campañas realizadas por parte de algunas instituciones; así como entender la existencia real de una posible oportunidad de negocio a la hora de ampliar y diversificar sus públicos con este grupo de edad específico.

Se trata de un trabajo de reflexión en el que se han empleado datos estadísticos, así como otras fuentes secundarias pertinentes, que han permitido llegar a algunas deducciones originales. Asimismo, se han analizado proyectos y campañas desarrolladas por distintos museos permitiendo comprender algunas de las principales tácticas y estrategias para la atracción de este público. Partiendo de estos datos se intenta dar respuesta a las problemáticas arriba mencionadas, realizando una original aportación de los usos y costumbres actuales de la generación $\mathrm{K}$ en el consumo de arte y museos.

\section{Desarrollo de la Innovación}

La industria cultural viene cambiando mucho en los últimos años, no sólo en lo que respecta a sus procesos de producción y distribución, sino también los hábitos, los gustos y las prácticas culturales. Un cambio importante viene del acceso a internet y a las tecnologías de la comunicación, que alojan gran parte de los contenidos y bienes del sector. No obstante, la COVID-19 acelera una transformación digital crucial y más que necesaria en el sector del arte y de los museos, lo que representa a priori una gran oportunidad para el sector.

Los museos que se han visto obligados a cancelar su agenda, con motivo de la crisis de la Covid-19, trasladan algunas de sus actividades a la web y redes sociales lo que produce, con el tiempo, una explosión de contenidos de estas organizaciones. Prueba de ello es que después de tres semanas de cierre al público, el $80 \%$ de los museos ya había incrementado su actividad online según la encuesta sobre el impacto de la situación de la COVID-19 en los museos de

\footnotetext{
${ }^{1}$ El término generación K fue acuñado por Noreena Hertz ${ }^{1}$, procede de Katniss Everdeen, la heroína de Los juegos del hambre.
} 
Europa realizada por la Red de Organizaciones de Museos Europeos (NEMO). Si bien es cierto que muchas de estos contenidos ya existían, su oferta se amplió o/ y se dio a conocer con más fuerza, durante los meses de confinamiento, mediante canales digitales. En lo que respecta a concretamente a España, un $73 \%$ de las organizaciones culturales ha puesto en marcha alguna actividad digital en estos meses (Fundación Contemporánea, 2020). Por otro lado, casi el $80 \%$ de los museos ha cambiado las tareas del personal para adaptarse a las necesidades de generación y gestión de esta actividad online, el 30\% ha sido reasignado al equipo digital y un $16 \%$ ha aumentado su presupuesto para el diseño y ejecución de actividades digitales (NEMO).

Debido a las actividades implementadas y a los ajustes realizados en lo relativo a los recursos económicos y humanos, más del 40\% de los museos encuestados notaron un aumento en las visitas online. Los incrementos semanales, durante el mes de abril 2020, oscilaron entre un 10-20\% hasta un 200\%, según han indicado el 5\% de las instituciones consultadas (NEMO, 2020).

Ejemplos más concretos y cercanos de esta tendencia son los más de dos millones de usuarios que han visitado la web del Museo el Prado $^{2}$, desde el inicio del confinamiento general ordenado por el Gobierno de España en la noche del 14 de marzo 2020. El número de usuarios de la web de la pinacoteca se ha incrementado en un $258 \%$ respecto a febrero, hasta alcanzar las 2.184.000 (Museo del Prado, 2020). Asimismo, se ha producido un incremento en la visualización de sus contenidos tanto en Instagram como en Facebook, aumentando un 134\% y un $124 \%$ respectivamente. Concretamente, con respecto a los stories de la cuenta de Instagram del Museo, las visitas en abril 2020 superaron en un 3.710\% las de febrero 2020, hasta llegar a 1.581.500. Otro ejemplo es el incremento del $400 \%$ en las visitas a la web del Museo Reina Sofía ${ }^{3}$, además de una intensificación en la relación con otros públicos a través de sus redes sociales, experimentado un crecimiento promedio de un $300 \%$, en lo que se refiere tanto a visitas como a interacciones (Europa Press, 2020).

Pero, por lo que parece, no solo ha aumentado el tráfico en web y redes sociales, durante los primeros meses del 2020, sino que también se han generado nuevos públicos, nuevas generaciones de jóvenes que, aunque nativos digitales y muy adaptados al consumo digital y audiovisual en streaming, se han interesado por la cultura y por la visita virtual a museos.

\footnotetext{
2 https://www.museodelprado.es

3 https://www.museoreinasofia.es
} 
Según Ford W. Bell, presidente de la Alianza Americana de Museos "El cambio generacional es algo a lo que muchos gestores culturales les está preocupando enormemente, la división que separa a los jóvenes de las organizaciones artísticas parece ser más profunda. ". Teniendo en cuenta la actual crisis de audiencias que padecen muchas instituciones culturales, esta parece representar una excelente oportunidad para generar, capitalizar y fidelizar nuevos públicos.

\section{Resultados}

Los museos europeos han detectado un crecimiento de sus audiencias jóvenes en sus canales digitales. El caso de TikTok es paradigmático ya que, a pesar del veto que Donald Trump quería imponer a esta red social, ha sido la aplicación más descargada y popular de 2020 y ha sido una herramienta poderosa que ha revolucionado el tráfico de algunos museos. El caso concreto de la Galería Uffizi ilustra muy bien esta disrupción. La institución abrió su cuenta en marzo del 2020 y ya han generado más de 80.000 seguidores $^{4}$, de los cuales un $69 \%$ tiene entre 13 y 24 años. No obstante, lo más llamativo es que en los meses de junio, julio y agosto del 2020 entre el 34\% y el 45\%, de todos los visitantes a la Galería Uffizi, tenía menos de 25 años, frente al 18\%-28\% del verano del 2019 (El País, 2020). Por lo que parece, se está generando un cambio radical de registro narrativo de los contenidos, que se salta por primera vez a los intermediarios, y la obra dialoga directamente con el usuario. A pesar de tratarse de videos poco tradicionales, divertidos y breves no están descuidados, ni están improvisados ni mucho menos sin un control o curaduría, el proyecto de TikTok en la Galería Uffizi está dirigido por un equipo interdepartamental compuesto por 12 personas. Por otro lado, es muy interesante el efecto que ha tenido la microinfluencer Martina Socrate ${ }^{5}$, con 1.1 millón de seguidores, que condujo a un crecimiento del perfil de esta institución de un $50 \%$. En cuánto al Museo del Prado, su cuenta de TikTok cuenta con más de 140,000 seguidores, entre los que se encuentra una mayoría de usuarios de 18 años o menos, según la propia institución.

Estos jóvenes los podemos segmentar en millennials o generación Y (quienes hoy tienen entre 22 y 42 años), sobretodo eco-millennials, y centennials o generación $\mathrm{Z}$ o K (de cero a 21 años), en conjunto representan más de 4,400M, 2.000M y $2.400 \mathrm{M}$ respectivamente, lo que equivale a un 27\% y un 32\% de la población mundial (Pew Research Center, 2019).

\footnotetext{
${ }^{4}$ https://www.tiktok.com/@uffizigalleries?lang=en

${ }^{5}$ https://www.tiktok.com/@martinasocrate?lang=en
} 
Aunque en su conjunto ambos grupos de edad son, y serán, la generación principal de consumidores culturales, patronos y filántropos, me gustaría profundizar específicamente en la generación K. En 2020, todas las generaciones han tenido que cambiar rápidamente la forma en la que consumir, socializar, aprender, divertirse e incluso empoderarse pero la generación que mejor y más rápido se ha adaptado a estos cambios ha sido, precisamente, la Generación K. Por otro lado, es un grupo de edad al que se la conoce muy poco, representa el futuro del consumo masivo y presenta un reto más que interesante para el sector cultural.

La generación K es el grupo de edad más grande en la historia de Estados Unidos y se estima que tiene un poder adquisitivo de $\$ 44$ mil millones (Wertz, 2019), representando en EEUU un $40 \%$ de los consumidores. El $83 \%$ de estos jóvenes duerme junto a su smartphone, lo consultan 45 veces al día y en Estados Unidos la edad media a la que poseen su primer móvil inteligente apenas supera los 10 años. Este dispositivo representa un canal más en su proceso de compra y consumo, que es omnicanal, holístico y muy complejo, con un particular, y todavía no entendido $100 \%$ customer journey. El término generación $\mathrm{K}$ fue acuñado por Noreena Hertz y procede de Katniss Everdeen, la heroína de Los juegos del hambre interpretada por Jennifer Lawrence. Como ella, estos jóvenes, entre 13 y 20 años, sienten que viven en un mundo en permanente conflicto. Una sociedad violenta, distópica e injusta ante la que deben reaccionar. Ejemplo de ello es la pandemia y sus efectos colaterales, así como la disrupción que está generando la tecnología y, en especial, la inteligencia artificial que redefinirá muchas de las profesiones que hoy en día conocemos. Según Noreena Hertz a la generación $\mathrm{K}$ no les interesan los valores que proyecta una marca, un gobierno, un autor o una exposición sino las conclusiones a las que llegan bebiendo de distintas fuentes. Es una generación que busca autenticidad en le mensaje y conexiones emocionales. Otro aspecto importante, es la facilidad que tienen para moverse entre la alta y baja cultura, entre el arte, el diseño y la moda, buscando, por lo tanto, experiencias multidisciplinares.

Así como los Médici, los famosos mecenas del arte florentino durante el Renacimiento italiano, pagaron a artistas para representar a los miembros de la familia, los miembros de la generación $\mathrm{K}$ usan sus identidades en las redes sociales como homenaje a sí mismos y como formas de significar y representar el mundo que los rodea. A los miembros de este grupo de edad les gusta ser individuales y únicos, con una manera muy personal de pensar y para quienes su propia imagen ocupa un lugar primordial a la hora de expresarse y el selfie parece ser la manera de canalizarlo. Asimismo, podemos identificar la necesidad de obtener una experiencia más interactiva, en lugar de pasiva, con las artes. Requieren, al mismo tiempo, 
experiencias en las que se ofrezca la misma personalización y usabilidad de sus experiencias virtuales, en las que puedan publicar, comentar y dar me gusta al instante. El Museo de Arte Moderno de San Francisco han demostrado estar a la altura del desafío de la generación K, mientras cumplen impecablemente con su misión artística y educativa. Esta institución, mediante la alianza con empresas de tecnología de Silicon Valley, se ha focalizado en generar experiencias participativas en algunas de sus exposiciones con realidad virtual y realidad aumentada. Un par de ejemplos, en ese sentido, son la exposición de René Magritte ${ }^{6}$ y el proyecto Self Composed ${ }^{7}$, que contó con un espacio interactivo en el que a los visitantes se les permitía re imaginar, reconstruir y crear sus propios selfies. Estas acciones han permitido atraer a más de un $45 \%$ de asistentes menores de 35 años, según fuentes del museo.

Por otro lado, en lo que respecta a una posible oportunidad de negocio, los resultados de un informe recientemente elaborado por MasterCard España reflejan que el 40\% de los clientes encuestados gasta cada vez más dinero en experiencias virtuales, el 78\%, se ha suscrito a un servicio online de streaming y más de una cuarta parte (28\%) ha visitado virtualmente un museo. Por otro lado, según el informe, The Economics of Creativity, elaborado por The Economist Intelligence Unit, un $41 \%$ de los británicos ha consumido cultura digital, durante los meses de confinamiento, y un 78\% estarían dispuestos a pagar, también durante la post pandemia. Más de mitad (57\%) de los jóvenes (entre 14 y 25 años) del Reino Unido estaría dispuesto a pagar por actividades culturales online, siendo los museos y galerías los protagonistas (78\%). Asimismo, un 83\% de esta generación, en Reino Unido, que ha consumido cultura online durante el confinamiento, estaría dispuesta a pagar más por experiencias, en persona, una vez transcurra la pandemia. Se trata de un grupo de edad que, al igual que los millennials, está dispuesto a pagar un mayor valor por experiencias o productos alineados con su filosofía, proceso en el que predomina la unicidad versus la exclusividad, así como la influencia y el potencial impacto provocado (Pew Research Center, 2019). No obstante, en lo que respecta a España la situación es diferente ya que, según el estudio Consulta Urgente, del 2020, la mayoría de las instituciones culturales españolas (62\%) ha ofrecido, durante el confinamiento, contenidos digitales gratuitos y tan solo un 11\% ha promocionado la venta online de sus productos culturales previos y un $9 \%$ ha conseguido

\footnotetext{
${ }^{6}$ https://www.stereolabs.com/blog/zed-magritte-sfmoma

${ }^{7}$ https://www.behance.net/gallery/49165305/Self-Composed
} 
nuevos ingresos lanzado nuevos productos de pago, por lo que es difícil medir esta posible oportunidad de negocio para las instituciones culturales.

Un ejemplo más concreto es el de TATE Collective ${ }^{8}$ que consiste en un programa de fidelización dirigido exclusivamente a jóvenes entre 16-25 años, el cual presenta muchas ventajas $^{9}$, entre las que se encuentran el acceso a exposiciones y descuentos dentro del museo, entre otras. Asimismo, se les da la posibilidad de involucrarse activamente en el diseño de la programación de la TATE. Este posibilidad de empoderamiento, pertenencia y co-producción está muy ligado a los valores de este público, al que le gusta crear su propio arte, ser sus propios comisarios y además actúan como distribuidores de sus creaciones. Uno de los objetivos principales, a largo plazo, de este programa es desarrollar una relación sostenible con audiencias muy comprometidas que pasarán eventualmente por todos los estadios del involucramiento humano y económico con el museo. En 2019 consiguieron facturar casi un millón de libras mediante este programa de amigos, con una tendencia creciente desde su creación.

\section{Conclusiones}

Del análisis realizado podemos deducir que esta pandemia ha actuado como un acelerador de partículas haciendo que los museos y sus colecciones ocupen un espacio de gran relevancia en nuestro tiempo libre a golpe de click. No solo se ha generado un enorme interés por los contenidos disponibles en los canales digitales de los distintos museos y centros de arte, sino que también se han generado nuevos públicos, nuevas generaciones de jóvenes que se han interesado por la cultura y los museos. Tanto los estudios de The Economist Intelligence Unit, como el incremento en un $3.710 \%$ de las visitas a los stories de Instagram del Museo del Prado, compuestas según el Globalwebindex por un $80 \%$ de personas entre 16 y 30 años, así como el interés y la correlación existente entre los usuarios de TikTok y el incremento de las visitas al espacio físico de la Galería Uffizi, entre personas de entre 13 y 24 años, nos permite entender el interés incipiente que existente por parte no solo de los jóvenes sino más concretamente por parte de la generación $\mathrm{K}$.

\footnotetext{
${ }^{8}$ https://www.tate.org.uk/tate-collective/we-are-the-future

${ }^{9} \mathrm{https} / / /$ shop.tate.org.uk/collective-register
} 
Parece que este grupo de edad ha convertido el video en su formato predilecto y ha aplicado la cultura de lo efímero al contenido, ha reemplazado las palabras por emojis y ha hecho de la creatividad su sello personal. El resultado de estos cambios se ha visto reflejado en el éxito de TikTok y de los principales museos de Europa, convirtiendo al usuario en protagonista de la acción: ya no solo consume el contenido, sino que también lo genera. En este sentido, reformular la estrategia de comunicación digital del museo implica abrirlo a nuevos canales y a nuevas formas de expresión que permitan llegar a este público y fomenten su participación en la creación de contenidos y convertirlos en prosumidores.

Teniendo en cuenta la actual (y problemática) división que separa a los jóvenes de las organizaciones culturales más tradicionales, esta nueva ventana parece representar una clara oportunidad para conectar con nuevas audiencias, especialmente jóvenes (y, por tanto, del futuro). Se hace, asimismo, evidente, explorar canales adicionales y estrategias que permitan generan ingresos mediante el diseño de nuevos modelos de negocio bien alienados con los usos y costumbres de estas nuevas y jóvenes audiencias. Sobretodo teniendo en cuenta el gran mercado que representan $(+2,4000$ millones de personas) y su predisposición para pagar por actividades culturales incluso después del confinamiento, según el estudio de The Economist Intelligence Unit.. Aferrarse a esta oportunidad parece ser una de las claves de la supervivencia de la industria creativa.

Se está configurando, en estos momentos, un mercado sustancial con nuevas características, necesidades y demandas, nuevas interacciones con sus dispositivos a la hora de comprar y disfrutar de las experiencias, diferencias en el engagement, así como preferencias con respecto a contenidos o en relación a la propensión de pagar por los mismos, que conviene conocer en profundidad por las repercusiones y transformaciones que estará exigiendo, muy pronto, a los museos en pro de ser relevantes y de generar relaciones sostenibles con sus públicos actuales y futuros. 


\section{Referencias}

AMERICAN ALLIANCE OF MUSEUMS. United States May Lose One-third of All Museums, New Survey Shows. $<$ https://www.aam-us.org/2020/07/22/united-states-maylose-one-third-of-all-museums-new-survey-shows/> [Consulta: 5 de octubre de 2020]

FARAGO, J (2020). Now Virtual and in Video, Museum Websites Shake Off the Dust.

New York: New York Times. <https://www.nytimes.com/2020/04/23/arts/design/bestvirtual-museum-guides.html> [Consulta: 4 de octubre de 2020].

FUNDACIÓN CONTEMPORÁNEA. Consulta Urgente.

$<$ https://fundacioncontemporanea.com/wpcontent/uploads/2012/10/Observatorio_de_la _Cultura_URGENTE_.pdf $>$ [Consulta: 10 de octubre de 2020]

GARCÍA VEGA, M.A. (2016). Los 'millennials' y los 'centennials', dos generaciones que valen 19 billones. Madrid: El País

$<$ https://elpais.com/economia/2016/10/20/actualidad/1476985002_101940.html $>$ [Consulta: 5 de octubre de 2020].

GRIJOTA, S. (2020). Adiós academia, hola TikTok: la estrategia sin complejos con la que la Galería Uffizi ha duplicado los visitantes jóvenes en tres meses. Madrid: El País. $<$ https://elpais.com/icon-design/2020-10-18/adios-academia-hola-tiktok-la-estrategiasin-complejos-con-la-que-la-galeria-uffizi-ha-duplicado-los-visitantes-jovenes-en-tresmeses.html> [Consulta: 4 de octubre de 2020].

HERTZ, N (2015). Generation K: what it means to be a teen. London: Financial Times. $<$ https://www.ft.com/content/1642f9d2-e3ac-11e4-9a82-00144feab7de $>$ [Consulta: 6 de octubre de 2020].

KOTLER, P., KOTLER, N. (2001). Estrategias y marketing de museos. Barcelona: Ariel. MINISTERIO DE CULTURA Y DEPORTE DE ESPAÑA. Visitas a las webs.

$<$ https://www.culturaydeporte.gob.es/cultura/museos/museos-cifras/visitas-web.html> [Consulta: 6 de octubre de 2020]

MUSEO DEL PRADO Almost 2 million people visit the Prado during the confinement. $<$ https://www.museodelprado.es/en/whats-on/new/almost-2-million-people-visit-theprado-during/8a4e5d5a-4006-6108-9c92-c2d9b4415101> [Consulta: 5 de octubre de 2020]

NETWORK OF EUROPEAN MUSEUM ORGANISATIONS. Survey on the impact of the COVID-19 situation on museums in Europe. 
$<$ https://www.nemo.org/fileadmin/Dateien/public/NEMO_documents/NEMO_COVID19_ Report_12.05.2020.pdf [Consulta: 10 de octubre de 2020]

THE ECONOMIST INTELLIGENCE UNIT. The Economics of Creativity.

$<$ https://economicsofcreativity.economist.com/> [Consulta: 2 de octubre de 2020]

UNESCO. Museums around the world in the face of COVID-19.

$<$ https://unesdoc.unesco.org/ark:/48223/pf0000373530?fbclid=IwAR0JGX8DmJZUM1

WPK7mMF8FDx4_x8FDJIYEOy0YO6jH63mBTjtQhhP_yN1w> [Consulta: 2 de octubre de 2020] 